Research Article

\title{
The Impact of COVID-19 on the Dependence of Chinese Stock Market
}

\author{
Xianbo Wu $(\mathbb{D})$ and Xiaofeng Hui \\ School of Management, Harbin Institute of Technology, Harbin 150001, China \\ Correspondence should be addressed to Xiaofeng Hui; xfhui@hit.edu.cn
}

Received 30 January 2021; Accepted 6 May 2021; Published 20 May 2021

Academic Editor: Lijun Pei

Copyright (C) 2021 Xianbo Wu and Xiaofeng Hui. This is an open access article distributed under the Creative Commons Attribution License, which permits unrestricted use, distribution, and reproduction in any medium, provided the original work is properly cited.

By calculating the mutual information of stock indexes of 10 primary industry sectors in China, this paper analyzes the dependence relationship among Chinese stock sectors during the COVID-19 and the dynamic evolution of the relationship by using the sliding window method. According to the actual situation of the development of COVID-19 in China, the samples were divided into three stages, namely, calm period, pandemic period, and post-pandemic period. The results show that the dependence relationship among Chinese stock sectors is significantly enhanced in the pandemic period, but it decreases in the post-pandemic period and the dependence structure is similar to that in the calm period. The industrials sector is most closely connected with other sectors in the pandemic period. The information technology sector and telecommunication services sector maintain strong dependence in the three periods and share little contact with other sectors. In the pandemic period, the dependence between the consumer staples sector and other sectors is significantly enhanced, and consumer staples sector and health care sector maintain a strong dependence. From the results of the sliding window, the Chinese stock market is sensitive to the impact of COVID-19, but the duration of the impact on the dependence among the stock sectors is not long.

\section{Introduction}

At the beginning of 2020, the outbreak of COVID-19 caused global panic. It is reported by WHO that up to September 1st, 2020, there have been 25,327,098 confirmed cases of COVID19 , including 848,255 deaths globally. Since the outbreak of COVID-19, in order to effectively curb the spread of the pandemic, many countries have implemented a series of necessary measures, including shutdown of the production and business and home isolation. The implementation of these strict measures has brought a great impact on the economic development and the operation of enterprises. At present, there have been some studies about the impact of COVID-19 on world economic and social development. Studies have found that even in the early stages of COVID-19, its impact on the real economy has already been reflected [1], the pandemic has negatively affected the trade, tourism, and transportation, and it increased the unemployment rate [2]; even some studies showed that the global spread of COVID-19 had made a similar impact to an economic crisis [3].

COVID-19 not only has a great impact on economic and social development, but also has an influence on the operation of financial markets. Some studies showed the impact of COVID-19 had been reflected in the return of financial markets [4-8], the volatility of financial markets [9-12], and the risk contagion among financial markets [13]. Baig et al. investigated the impact of COVID-19 on the liquidity and volatility of the stock market and found that the increase in confirmed cases and deaths due to COVID-19 had significantly aggravated the lack of liquidity and volatility in the market, and strict closure measures had also deteriorated the liquidity and stability of the market [14]. Rizwan et al. studied the banking systemic risk in eight major countries which were seriously affected by COVID-19; it was found that the financial systemic risk of each country increases significantly during the pandemic period [15]. 
In addition, some studies focused on the performance of the stock in different sectors or different countries. Mazur et al. found that the return of healthcare, food, natural gas, and software sectors performed well during the pandemic; however, crude petroleum, real estate, entertainment, and hospitality sectors fell sharply, and they had great volatility [16]. Shehzad et al. compared the impact of COVID-19 on the stock market with that of global financial crises; it was found that the European and American stock markets were more seriously affected by COVID-19, and the pandemic had blocked the global economic communication and caused a financial crisis [17].

Clearly analyzing the interdependence structure of the stock market is not only of great significance for investors to diversify investment and build investment portfolios during the pandemic, but also is conducive to risk management of the financial market by the regulatory authorities. In recent years, there has been a certain amount of researches on the subject of interdependence among stock markets [18-25]; in these studies, GARCH model, Copula model, Granger causality test, DCC model, and other methods are used to study the interdependence structure between various stock sectors in different countries.

Existing research on the interdependence structure of the stock markets has shown that the study could effectively identify which sector plays a more important role in the national economy and could also provide new opportunities for investors to carry out a reasonable portfolio of assets [26]. However, there are very few studies on the interdependence structure of the stock sectors during the special period of COVID-19. China is the first country to carry out the preventive measures toward COVID-19 and the initial success has been achieved, and now China's fight against the pandemic has entered a new normal period. In addition, more and more investors hope to obtain higher profits and reduce investment losses as much as possible through scientific investment methods. Diversification investment, which distributes assets to the stocks of listed companies belonging to different sectors, has become a very popular way for investors. The essence of diversification is to prevent the investment loss caused by investing the closely dependent assets. COVID-19 is another great recession of economic and social development since the global financial crisis in 2008; to clarify the structure and changes of dependence among various sectors in this crisis is a very useful guidance for investors to diversify their investment during public health events like COVID-19. In addition, the study of the dependence of Chinese stock market during the period of COVID-19 also has a strong guiding role for investors from other countries in the world to invest in their own stock market. Therefore, it is of great significance to study the interdependent structure of Chinese stock sectors and its changes during COVID-19.

\section{The Mechanism of Dependence of Stock Market and Methodology}

2.1. The Mechanism of Dependence of Stock Market. In recent years, with the rapid development of information technology, investors can easily and quickly carry out stock trading.
It is the common pursuit of investors to obtain more profits and reduce the risk as much as possible. Therefore, they often analyze the future market trend according to the current situation of the market and then formulate corresponding investment strategies according to their expectations. When a large number of investors adopt similar investment strategies in different markets, the emergence of the stock market dependence is possible.

Kodres and Pritsker proposed a rational expectation model of asset prices to explain the contagion phenomenon in financial markets. They found that market shocks can pass from one market to another by the way investors adjust their portfolios [27]. Barberis et al. proposed that investors' classified investment and investment preference are also important reasons for stock dependence [28]. Classified investment refers to that when some investors make investment decisions, because there are a large number of stocks in the market, it will cost a lot to examine them one by one. Therefore, investors often first divide the stocks in the market into several different categories, such as by the industry, theme, and other characteristics of the stocks, and then allocate funds according to the categories. Therefore, if some investors adopt similar classified investment strategies, they introduce the same factors into the same kind of stocks when they transfer funds from one sector to another, even if the cash flows of these stocks are not related, and then promote the emergence of the dependence relationship between them. Investment preference means that due to transaction costs, international trading restrictions, lack of information, and limited attention, investors often choose only a part of the available stocks to invest. When investors' risk aversion, mood, or liquidity needs change, they adjust their funds within the range of preferred stocks, which leads to the introduction of common factors in these preferred stocks, resulting in the dependence between these stocks.

Investors' decision-making is often influenced by other investors' behavior, which leads to herd behavior of imitating others' investment decision-making, overrelying on public opinion and ignoring their own information. This is because there is a large amount of uncertain information in the market. It is not only difficult to collect and process them, but also requires a high cost, which individual investors cannot afford, prompting them to follow other investors' strategies. Herd behavior also exists in professional investment managers. This is because, due to the complexity of the market, even professional investment managers cannot guarantee the correct investment strategy. When they lack confidence in their strategy, the best way is to choose to be consistent with the strategy of other investment managers. In this way, we can avoid the damage to our reputation caused by our different strategies [29]. The similarity of investors' strategies will also enhance the dependence of stocks in the market. For example, when investors buy a lot of stocks, the prices of these stocks may rise at the same time. When other stocks are sold by a large number of investors at the same time, the prices of these stocks may fall at the same time, and herd behavior promotes the transmission of information between markets [30]. 
2.2. Mutual Information. At present, most of the research methods on the dependence of the stock markets are still based on linear assumptions or based on a specific model and parameters. For example, the Pearson correlation coefficient method, which is the most basic and most commonly used in calculating the dependence relationship, could only measure the linear relationship between variables, instead of effectively measuring the nonlinear relationship. Besides, the Granger causality test method and multivariate GARCH models are also only suitable for linear condition. However, a lot of existing studies have confirmed that there are a large number of nonlinear conditions in the financial markets. Compared with the above methods, the Copula method could be used in the situation of nonlinearity; however, the model selection and parameter setting are required in this method, and different model selections and parameter settings will affect the accuracy of the empirical results. Therefore, it is particularly important to choose a method that can be used to measure the dependence under nonlinear condition, at the same time avoiding the influence of the model selection and parameter setting. With the development of entropy theory and the expansion of its application, methods such as mutual information have been applied in a large number of financial market researches [31-36]. This method can well overcome the shortcomings of the abovementioned various methods. Therefore, this article will analyze the interdependence among Chinese stock sectors based on entropy theory and the method of mutual information.

The definition of information entropy was made by Shannon in the 1940s, and it could be used to measure the extent of uncertainty. According to the definition, for a random variable, the entropy of the variable can be expressed as follows:

$$
H(X)=-\sum_{x \hat{\mathrm{I}} \chi} p(x) \log p(x)
$$

where $\chi$ is the set of all states of the random variable $x$ and $p(x)$ is the probability of $x$ in the position of $\chi$.

For two random variables $x$ and $y$, the joint entropy between these two variables can be defined as follows:

$$
H(X, Y)=-\sum_{x \in X} \sum_{y \in Y} p(x, y) \log p(x, y),
$$

where $p(x, y)$ is the joint probability of $x$ and $y$.

For the given two variables $x$ and $y$, assuming that their respective marginal probability distributions and joint probability distribution are known, and they are, respectively, $p(x), p(y)$, and $p(x, y)$, the mutual information between these two variables can be expressed by the following formula:

$$
I(X, Y)=\sum_{x \in X} \sum_{y \in Y} p(x, y) \log \frac{p(x, y)}{p(x) p(y)} .
$$

According to formulae (1) and (2), the mutual information formula (3) can be rewritten as follows after calculation:

$$
I(X, Y)=H(X)+H(Y)-H(X, Y) .
$$

It can be seen from formula (4) that in terms of mathematical relationship, the value of mutual information of two variables can be expressed as the sum of the entropy of these two variables, subtracting the joint entropy from them. In terms of the theoretical sense, the mutual information of two variables means that if the information contained in one of the two variables has been mastered, to what extent, it will reduce the uncertainty of the other variable. The mutual information between two variables can be understood as the amount of information shared together; that is, if one of the two variables is known, and thus the uncertainty of the other variable can be reduced substantially, then it can be considered that this known variable contains a lot of information of the other variable.

2.3. Kernel Density Estimation. Estimating the probability density and distribution is the basis of empirical researches. At present, a large number of studies are based on certain assumptions, such as the assumption of the samples obeying the normal distribution. Under this assumption, the parameters are estimated. However, a large number of financial time series are not strictly subject to some single probability distribution. Therefore, the previous hypothesis that samples obey a certain distribution will lead to inaccurate parameter estimation and result in deviation. In order to overcome this problem, this paper uses the kernel density estimation method.

Let $\mathbf{U}=\left\{\mathbf{u}_{1}, \mathbf{u}_{2}, \ldots, \mathbf{u}_{N}\right\}$ be the sample with $d$ dimension; the kernel density estimation of the probability density function can be expressed by the following formula:

$$
\widehat{f}\left(u_{j}\right)=\frac{1}{N h^{d}} \sum_{i=1}^{n} K\left(\frac{u_{j}-u_{i}}{h}\right),
$$

where $h$ is the window parameter and can also be called bandwidth and $K(\cdot)$ is the kernel function with $d$ dimension.

In this paper, we choose the Gaussian kernel, which is commonly used in practice. Therefore, formula (5) can be rewritten as

$$
\widehat{p}\left(\mathbf{u}_{j}\right)=\frac{1}{N h^{d}} \sum_{i=1}^{n} \frac{1}{\sqrt{(2 \pi)^{d}|S|}} \exp \left(-\frac{\left(\mathbf{u}_{j}-\mathbf{u}_{i}\right)^{T} S^{-1}\left(\mathbf{u}_{j}-\mathbf{u}_{i}\right)}{2 h^{2}}\right),
$$

where $S$ is the value of covariance matrix [37].

The choice of bandwidth has a great influence on the estimation effect; according to the previous literature [38], the bandwidth is calculated by the following formula:

$$
h=\left(\frac{4}{d+2}\right)^{1 /(d+4)} N^{-1 /(d+4)} .
$$

The probability density of the samples can be obtained by kernel density estimation, and then the entropy formula can be obtained. 


$$
H(U)=-\frac{1}{N} \sum_{t=1}^{N} \log \widehat{p}\left(\mathbf{u}_{t}\right)
$$

Combined with formulae (4) and (8), we can get the formula for calculating the mutual information of two variables in this paper, as shown in the following formula:

$$
I(X, Y)=-\frac{1}{N} \sum_{t=1}^{N} \log \widehat{p}\left(x_{t}\right)-\frac{1}{N} \sum_{t=1}^{N} \log \widehat{p}\left(y_{t}\right)+\frac{1}{N} \sum_{t=1}^{N} \log \widehat{p}\left(x_{t}, y_{t}\right) .
$$

\section{Data}

This paper uses China's first-level industry sector index released by China Securities Index Co., Ltd as the research object. The industry sector index is shown in Table 1 , and the data are downloaded from the WIND database, which is a leading Chinese financial information provider, and the time range of the data is from August 1st, 2019, to August 28th, 2020. According to the different development status of COVID-19 in China, the interval is subdivided into three stages: calm period, pandemic period, and post-pandemic period. The calm period is from August 1st, 2019, to December 31st, 2019, the pandemic period is from January 1st, 2020, to April 28th, 2020, and the post-pandemic period is from April 29th, 2020, to August 28th, 2020. The above time period is divided according to the actual development situation of COVID-19 in China; COVID-19 was first reported by Chinese authorities on December 31st, 2019, to the World Health Organization [6], and according to the White Papers of Fighting COVID-19 China in Action, from April 29th, 2020, prevention and control of COVID-19 in China has been normalized, and the efforts have been made to promote the resumption of work, production, and education.

According to the experience of previous literatures, this paper calculates the logarithm return of each index according to the following formula:

$$
R(t)=\ln P(t)-\ln P(t-1),
$$

where $p(t)$ and $p(t-1)$ are the daily closing price of the regional index on dates $t$ and $t-1$, respectively and $R(t)$ is the logarithmic return of the regional index on date $t$.

It can be seen from the statistical characteristics of the returns of each sector in Table 2 that although some of the average returns of the ten sectors are positive and some are negative, they are very close to 0 . At the same time, the value of Kurtosis of each sector is greater than 3, which indicates that the distribution of their return rate is steeper than the normal distribution. Moreover, the results of Jarque-Bera test also reject the original hypothesis that it follows normal distribution, so it can be considered that the return of each sector does not follow normal distribution. ADF test results are significantly rejected by the original hypothesis of the existence of unit root, so it can be considered that the logarithmic returns of these markets are stable.

\section{Results and Discussion}

Firstly, we calculate the mutual information among the returns of 10 industry sector indexes, and each sector index is regarded as a node. When the mutual information value between any two sector indexes is calculated, the value is taken as the weight between the two nodes. Therefore, if the value of the weight between two nodes is large, it indicates that the mutual information between the two industry sector indexes represented by these two nodes is large and the correlation is strong; however, if the value of the weight between two nodes is small, it indicates that the mutual information between the two industry sector indexes is small and the correlation is weak.

By calculating the mutual information among the returns of 10 industry sector indexes in China and taking the value as the edge weight, the dependence relationship network of industry sector stock market in China is finally constructed. Figures 1-3 show the colormap of the dependence network of indexes in the calm period, pandemic period, and post-pandemic period, respectively. In the colormap, the numbers on the horizontal and vertical axes represent the 10 sector indexes in Table 1, and the color of the squares of coordinate $(X, Y)$ represents the weight between the two sector indexes numbered $X$ and $Y$. In order to facilitate the comparison of the changes of the interdependence among the 10 sectors in the three periods, this article adjusts the range of the color bars to the same one, $0 \sim 1.6541$, because the value of 1.6541 is the largest mutual information value among the industry sectors in the three periods and the maximum value appears in pandemic period.

It can be seen from Figure 1 that in calm period, the mutual information value among the 10 sectors is generally relatively small, especially the consumer staples sector (no. 5) and health care sector (no. 6). These two sectors have the smallest mutual information with other sectors, which indicates that they are less dependent on other sectors at this time. First of all, the enterprises in these two sectors, consumer staples sector (no. 5) and health care sector (no. 6), are not like the enterprises in industrials sector (no. 3), which have strong business or industrial connection with the enterprises in the other sectors. In addition, because the products provided by the enterprises in the above two sectors are necessities of people's life, their total demand is relatively stable in the calm period, and the change 
TABle 1: The names and codes of 10 industry sector indexes.

\begin{tabular}{lcc}
\hline No. & Index name & Code \\
\hline 1 & Energy & 000928 \\
2 & Materials & 000929 \\
3 & Industrials & 000930 \\
4 & Consumer discretionary & 000931 \\
5 & Consumer staples & 000932 \\
6 & Health care & 000933 \\
7 & Financials & 000934 \\
8 & Information technology & 000935 \\
9 & Telecommunication services & 000936 \\
10 & Utilities & 000937 \\
\hline
\end{tabular}

TABle 2: The statistics of 10 industry sector indexes.

\begin{tabular}{lcccccccc}
\hline No. & Mean & Std. Dev. & Max & Min & Skewness & Kurtosis & Jarque-Bera & ADF \\
\hline 1 & -0.0006 & 0.0133 & 0.0607 & -0.0805 & -0.4215 & 9.1562 & $421.4900^{* * *}$ & $-15.2299^{* * *}$ \\
2 & 0.0009 & 0.0151 & 0.0565 & -0.0951 & -0.8847 & 10.1636 & $594.3918^{* * *}$ & $-15.2248^{* * *}$ \\
3 & 0.0008 & 0.0144 & 0.0483 & -0.0954 & -1.1288 & 11.3717 & $820.7464^{* * *}$ & $-15.5941^{* * *}$ \\
4 & 0.0012 & 0.0158 & 0.0445 & -0.0934 & -1.2833 & 9.0477 & $471.1848^{* * *}$ & $-15.2167^{* * *}$ \\
5 & 0.0019 & 0.0160 & 0.0424 & -0.0818 & -1.1093 & 8.0413 & $331.1814^{* * *}$ & $-16.5019^{* * *}$ \\
6 & 0.0022 & 0.0158 & 0.0399 & -0.0705 & -0.6255 & 5.0340 & $62.2502^{* * *}$ & $-16.2852^{* * *}$ \\
7 & 0.0002 & 0.0151 & 0.0854 & -0.0829 & 0.0557 & 9.7765 & $501.4439^{* * *}$ & $-15.0286^{* * *}$ \\
8 & 0.0018 & 0.0222 & 0.0488 & -0.0985 & -0.8279 & 5.0344 & $75.1130^{* * *}$ & $-16.2218^{* * *}$ \\
9 & 0.0010 & 0.0215 & 0.0678 & -0.1020 & -0.4251 & 5.5435 & $78.5178^{* * *}$ & $-15.0867^{* * *}$ \\
10 & -0.0002 & 0.0101 & 0.0405 & -0.0806 & -1.5834 & 18.5162 & $2737.6930^{* * *}$ & $-16.4726^{* * *}$ \\
\hline
\end{tabular}

Note: ${ }^{* * *}$ denotes statistical significance at the $1 \%$ level.

of enterprise profitability is minor, so the investors have no motivation to adjust their investment portfolio or make up for investment losses in the calm period. Just like the previous elaboration on the mechanism of stock market dependence, it is very difficult for the asset portfolio without adjustment to transfer the information from one sector to another; therefore, these two sectors, consumer staples sector (no. 5) and health care sector (no. 6), are less dependent on other sectors at this time. It is worth noting that the mutual information value between the information technology sector (no. 8) and telecommunication services sector (no. 9) is very high, and it can be seen from Figures 2 and 3 that, in pandemic period and post-pandemic period, these two sectors always maintain a high dependence relationship since the software development and electronic equipment production provided by enterprises in the information technology sector (no. 8) and the local area network service and communication transmission equipment provided by enterprises in the telecommunication services sector (no. 9) show a lot of overlap in business with each other. For example, the Internet services and IT services provided by the listed companies in the information technology sector (no. 8) and the telecommunication operation services provided by the listed companies in the telecommunication services sector (no. 9) have business intersection, while the software development, electronic equipment, and semiconductors provided by the listed companies in the information technology sector (no. 8), and communications equipment provided by the listed companies in the telecommunication services sector (no. 9) also have business intersection. Therefore, the enterprises in the two sectors are often affected by the similar factors; as a result, the dependence between the two sectors is large. In addition, from the perspective of investors, when investing in one of the two sectors of information technology and telecommunication services, due to the limited energy and cost, the investors will also invest in the sector with similar products and services, and thus this investment behavior will inevitably increase the interdependence between the above two sectors.

Figure 2 shows the interdependence among Chinese stock industry sectors in pandemic period. It can be seen that the interdependence between the 10 sectors has generally improved at this time. This is consistent with the conclusions of the 2008 financial crisis and the 2015 Chinese stock market crash, when the interdependence among the stock markets increased significantly. During the period of uncertainty or volatility in the market, the investors always pay more attention to the performance of the stock market, increasing the trading operation or converting investment targets, these actions will increase the dependence between the stock markets [27, 39].

It is worth noting that the industrials sector (no. 3) and materials sector (no. 2) have the greatest dependence, and the consumer discretionary sector (no. 4) and financials sector (no. 7) also maintain the relatively close dependence relationship with the industrials sector. This conclusion is consistent with the performance of Chinese real economy in pandemic period. China is the first country to carry out the measures of prevention and control of the pandemic. In face of the sudden outbreak of COVID-19, almost all the cities in China carried out strict measures to prevent its spreading, including the establishment of mobile cabin hospitals and isolation facilities in community. As for the daily life of residents, with the popularity of logistics and takeaway, 
people have more choices for daily necessities including food, beverages, and personal products to purchase online, which has increased the demand for products and services provided by enterprises in the industrials sector. For the health care sector (no. 6), although it has also been widely concerned during the pandemic period, according to the results in Figure 2, the dependence between this sector and other sectors has not increased significantly, which could be interpreted that during the pandemic period, the investors pay more attention to consumer staples sector (no. 5) than health care sector (no. 6), and investors transfer a large amount of investment from other sectors to the consumer staples sector, which has significantly increased the dependence between consumer staples sector (no. 5), instead of health care sector (no. 6) and other sectors, and it is also consistent with the results in Figure 4. Comparing Figures 4(a) and 4(b), it can be seen that during the pandemic period, for the consumer staples sector (no. 5), which has the smallest node strength in the calm period, the dependence with other sectors has the fast growth in pandemic period, from the last one in the calm period to the fifth in the pandemic period; this indicates that a large number of sectors have enhanced the dependence with the consumer staples sector (no. 5) during the pandemic period, while the health care sector (no. 6) has the lowest node strength at this time. The dependence between information technology sector (no. 8) and telecommunication services sector (no. 9) is the same as that in the calm period, maintaining the highest dependence relationship with each other. For energy sector (no. 1) and utilities sector (no. 10), the dependence between them and other sectors has been improved, but the change is not significant.

Figure 3 shows the interdependence among sectors in the post-pandemic period. It can be seen that the interdependence of the 10 indexes during this period has significantly decreased compared with the pandemic period, but it is still higher than that in the calm period. It is worth noting that the structure of interdependence between the calm period and post-pandemic period is very similar. It illustrates that in the post-pandemic period the performance of stock markets and the behavior of investors are already similar to the state in the calm period, which can also prove that the fight against COVID-19 in China has achieved success preliminarily.

This paper then analyzes the node strength (NS) of each sector node in the industry sector indexes network in the three periods, respectively. The node strength reflects the sum of mutual information value of a certain sector index with all of the other ones, as shown in the following formula:

$$
N S_{i}=\sum w_{i j},
$$

where $W_{i j}$ is the mutual information between node $i$ and node $j$.

Figure 4 shows the node strength of each sector during the calm period, the pandemic period, and the post-pandemic period. The number on the horizontal axis is the sector number in Table 1, and the number on the vertical axis is the value of node strength. In order to facilitate comparison, the range of vertical axis in Figure 4 is adjusted to the same one. Comparing these three graphs, we can find that the dependence of sector indexes is highest in the pandemic period, and the node strength decreases in the post-pandemic period, which indicates that the dependence among sectors in this period is reduced but it is still higher than that in the calm period. During the three periods, the industrials sector (no. 3) has always maintained the maximum node strength, while the consumer staples sector (no. 5 ), which has the lowest node strength in calm period, has the fastest growth in pandemic period, from the last one in the calm period to the fifth in the pandemic period. Compared with the calm period, the dependence between consumer staples sector (no. 5) and other sectors has been significantly improved, since China implemented the strict measures in pandemic period, people could only stay at home even during the Spring Festival, so the daily consumer goods were paid more attention, such as food, beverages, etc., and they were provided by the enterprises in consumer staples sector. Therefore, enterprises in consumer staples sector have better performance in the pandemic period and attract the attention of investors. Therefore, investors will transfer their assets originally invested in other sectors to the consumer staples sector, which increases the dependence between the consumer staples sector and the other sectors.

Table 3 shows the changes of node strength of the sectors in the three periods. It can be seen that the standard deviation of consumer staples sector (no. 5) is the largest, indicating that the fluctuation of dependence between this sector and the other sectors is the largest one, which is also consistent with the explanation above. It is worth noting that the two sectors of information technology sector (no. 8) and telecommunication services sector (no. 9) not only maintain high dependence with each other, but also have little changes in dependence. Although the majority of Chinese people are in the state of home segregation during the pandemic period and more dependent on the products and services provided by companies in these two sectors, such as communicating with friends and relatives by use of QQ, WeChat, or other online tools, attending online classes and online meetings by use of Tencent Meeting, entertaining and relaxing by playing online games or TikTok, however in the great majority of households in China, the use of mobile phones, computers, and the Internet has already become quite popular. According to the statistical report on the development of China's Internet, by June 2019, the number of Internet users in China has reached 854 million, and the Internet penetration rate reaches $61.2 \%$, of which, the Mobile Internet users have reached $847 \mathrm{million}$, and the proportion of Internet users using mobile phones reaches $99.1 \%$. Therefore, the pandemic did not increase the demand for these products and services, and thus investors' attention has not shifted from other sectors to these two sectors, and the COVID-19 has little impact on the dependence of these two sectors among the other ones.

The above analyzes the interdependence network among the industry sector indexes from a static view, in order to explore the dynamic evolution of the dependence relationship among the sectors, the sliding window method is 


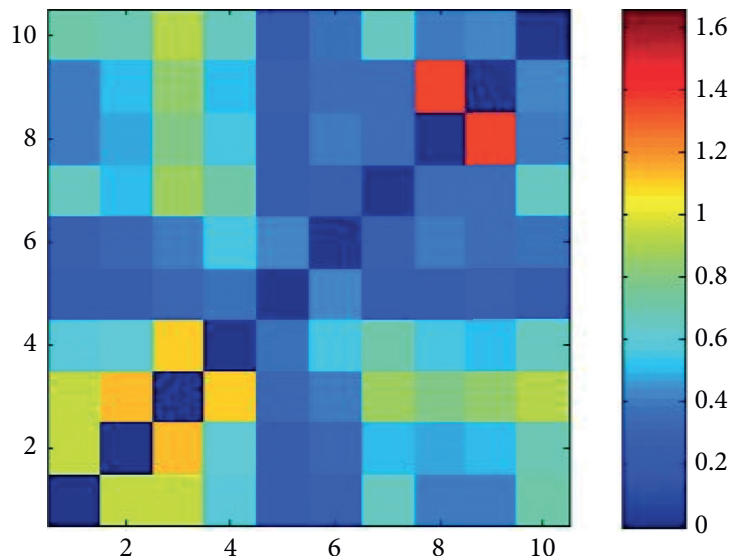

FIGURE 1: Colormap of industry sector indexes network in the calm period (the numbers on the horizontal and vertical axes represent the 10 sector indexes in Table 1).

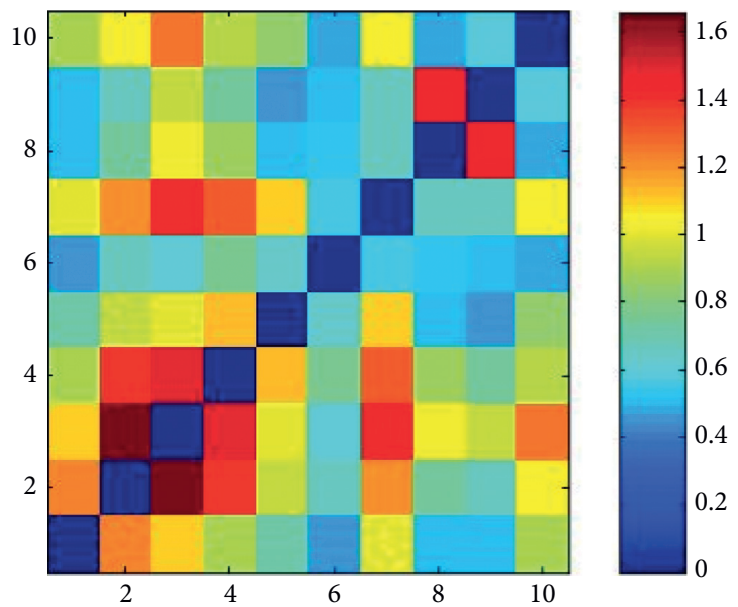

Figure 2: Colormap of industry sector indexes network in the pandemic period (the numbers on the horizontal and vertical axes represent the 10 sector indexes in Table 1).

used. In this article, the width of the sliding window is set as 50 days, and the sliding distance of each window is 5 days. The reason for the setting of window width and sliding distance is that it cannot only guarantee the number of samples required for each window, but also ensure sufficient comparisons.

According to the above setting, there are 43 sliding windows available, of which the 12th window enters into the pandemic period, and the 22nd to 26th windows are all within the pandemic period, after the 36th window, the pandemic period is no longer included.

In order to clearly observe the changes of the dependence of the 10 sectors in the pandemic period, Figure 5 shows the mean mutual information of all indexes that only cover the period of the pandemic (12th window to 36th window), and the 12th to 36th windows are renumbered from the 1st to 25th; the results are shown in Figure 5.

It can be seen from Figure 5 that at the beginning of entering the pandemic period, the mutual information among the sectors is small, but starting from the 4th window, the dependence increased rapidly, reaching the maximum value at the 11th to 13th windows, when the windows are completely in the pandemic period (the 10th to the 14th windows). After the 14th window, the mutual information between the sectors starts to decline. It can be learned from Figure 5 that the Chinese stock market is very sensitive to the impact of COVID-19; however, the impact of the pandemic does not last for a long time. This also shows that the Chinese measures for the prevention and control of the pandemic are effective and timely.

Table 4 analyzes, in the 43 sliding windows, with which one a certain sector maintains the strongest dependence relationship, in other words, which sector is most closely related to the certain one. For example, if the maximum weight of sector $A$ connects sector $B$, then we add 1 to the position of matrix $(A, B)$, and the initial value of position $(A$, $B)$ is 0 .

It can be found from Table 4 that the table is not symmetric, because the maximum weight edge of sector $A$ may connect sector $B$, while the maximum weight edge of sector $B$ may connect another sector $C$, which leads to asymmetry. It can be seen from the table that the industrials 


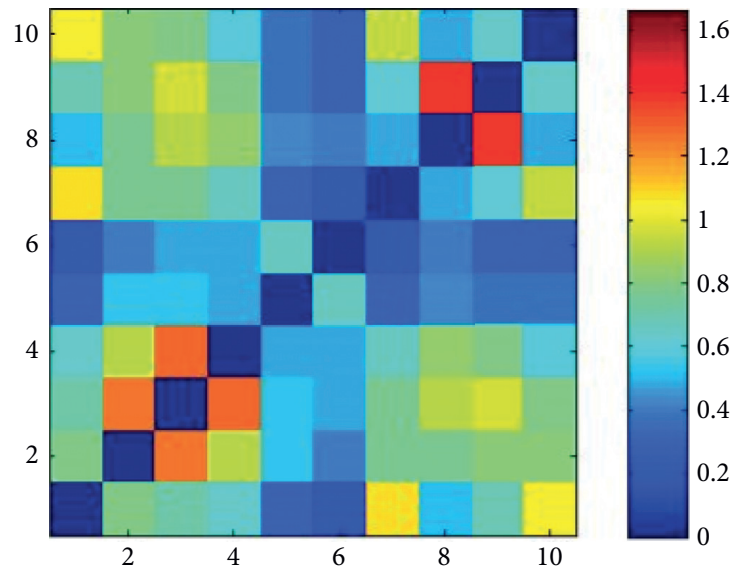

FIGURE 3: Colormap of industry sector indexes network in the post-pandemic period (the numbers on the horizontal and vertical axes represent the 10 sector indexes in Table 1).

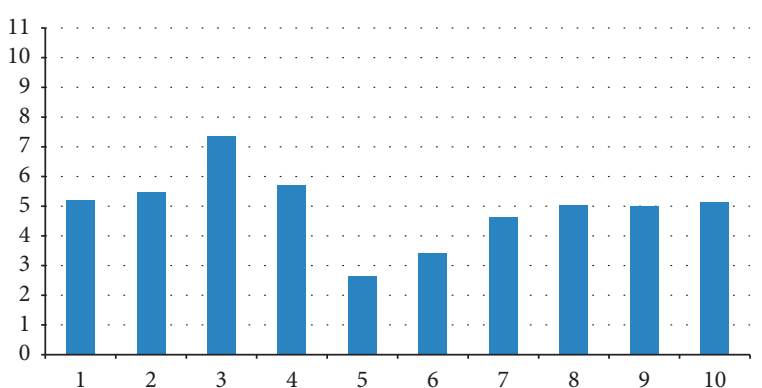

(a)

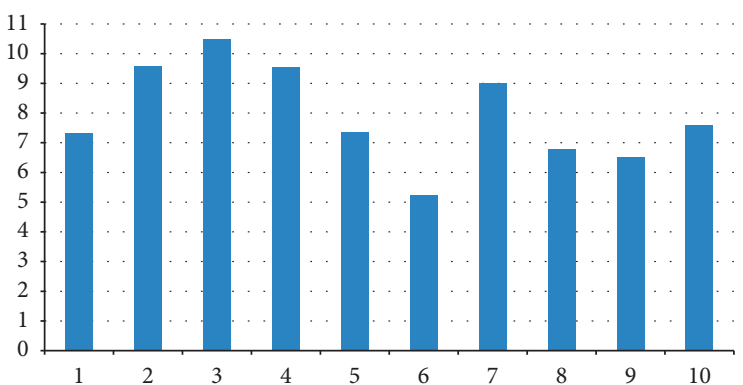

(b)

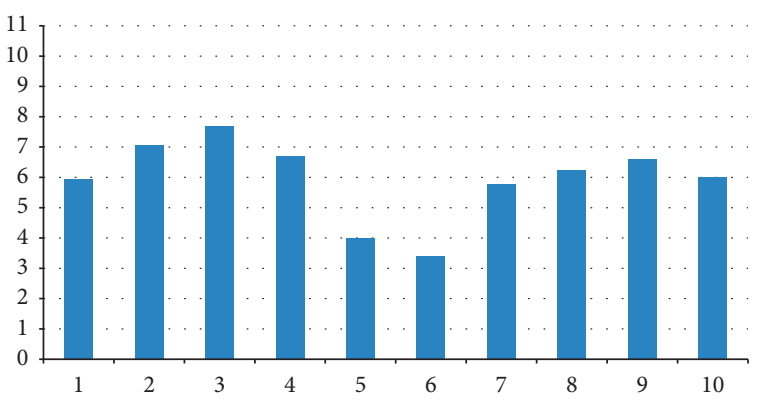

(c)

Figure 4: The NS value of the 10 sector indexes. (a) The calm period, (b) the pandemic period, (c) the post-pandemic period (the number on horizontal axis is the sector number in Table 1, and the number on vertical axis is the value of node strength).

TABLE 3: The standard deviation of NS of sectors in three periods.

\begin{tabular}{lcccccccccc}
\hline No. & 1 & 2 & 3 & 4 & 5 & 6 & 7 & 8 & 9 & 10 \\
\hline Std. Dev. & 0.8782 & 1.6927 & 1.4081 & 1.6380 & 1.9910 & 0.8649 & 1.8473 & 0.7373 & 0.7259 & 1.0199 \\
\hline
\end{tabular}

sector has the largest weight edge 142 times; among them, the largest weight edge is mainly given by the materials sector, consumer discretionary sector, and financials sector. For the information technology sector and telecommunication services sector, in all 43 windows, each of them is the largest edge of the other, which is consistent with the previous analysis. For the health care sector and consumer staples sector, the health care sector maintains the largest edge with the consumer staples sector in 18 windows, and the consumer staples sector keeps the largest edge with the health care sector in 21 windows. The largest edge obtained totally comes from each other. This shows that the two sectors are closely dependent. It can be understood that some investors who paid attention to the consumer staples 


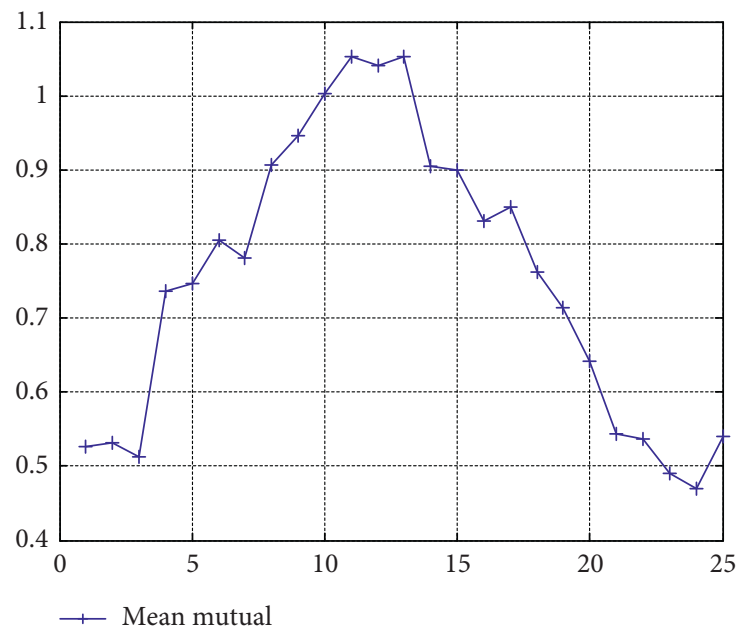

FIGURE 5: The mean mutual information among the 10 sector indexes in sliding window (the number on horizontal axis is the position of the window, and the number on vertical axis is the value of mean mutual).

TABLE 4: The total number of the strongest edges between the 10 sectors in sliding windows.

\begin{tabular}{lccccccccccc}
\hline No. & 1 & 2 & 3 & 4 & 5 & 6 & 7 & 8 & 9 & 10 \\
\hline 1 & 0 & 27 & 3 & 0 & 0 & 0 & 8 & 0 & 0 & 5 \\
2 & 2 & 0 & 40 & 1 & 0 & 0 & 0 & 0 & 0 & 0 \\
3 & 0 & 27 & 0 & 7 & 0 & 0 & 0 & 0 & 0 & 9 \\
4 & 0 & 6 & 37 & 0 & 0 & 0 & 0 & 0 & 0 & 0 \\
5 & 0 & 2 & 0 & 17 & 0 & 21 & 3 & 0 & 0 & 0 \\
6 & 0 & 1 & 0 & 21 & 18 & 0 & 0 & 0 & 1 & 43 \\
7 & 5 & 0 & 32 & 1 & 0 & 0 & 0 & 0 & 0 & 5 \\
8 & 0 & 0 & 0 & 0 & 0 & 0 & 0 & 0 & 43 & 0 \\
9 & 0 & 0 & 0 & 0 & 0 & 0 & 0 & 43 & 0 & 43 \\
10 & 5 & 0 & 30 & 0 & 0 & 0 & 8 & 0 & 0 & 0 & 43 \\
Sum & 12 & 63 & 142 & 47 & 18 & 21 & 19 & 43 & 44 & 43 \\
\hline
\end{tabular}

sector during the pandemic also paid attention to the health care sector, which increased the interdependence between the two sectors.

\section{Conclusions}

This article selects the 10 Chinese stock industry sector indexes from August 1st, 2019, to August 28th, 2020, as the research sample, the whole sample is divided into calm period, pandemic period, and post-pandemic period, according to the development of COVID-19 in China. By calculating the mutual information value among the sectors in each period, the dependence network is obtained, and then the sliding window method is used to study the dynamic evolution of the dependence structure. Through the abovementioned empirical analysis, this article draws the following conclusions.

Firstly, in the pandemic period, the dependence among Chinese industry sector indexes increases significantly compared with the other two periods. On the other hand, although the dependence in the post-pandemic period has dropped, it is still higher than that in calm period, and the structure of dependence between the post-pandemic period and the calm period is similar. This indicates that the pandemic prevention and control measures implemented in China have made great achievements, and people's production and business activities have basically returned to the previous state before the outbreak of COVID-19.

Secondly, the information technology sector and telecommunication services sector maintain a strong dependence with each other in all of the three periods and have little relationship with other sectors, which is related to the products and services provided by enterprises in these two sectors. The dependence between the consumer staples sector and other sectors is significantly enhanced in the pandemic period, and the health care sector maintained a strong dependence relationship with the consumer staples sector, indicating that during the pandemic period, the investors paid more attention to the consumer staples sector, and these investors also maintained a strong attention to the health care sector. In the pandemic period, the industrials sector is most closely connected with other sectors, especially these three sectors of materials sector, consumer discretionary sector, and financials sector, which is related to the role of enterprises in the industrials sector in the pandemic period. 
Finally, from the results of the sliding window, Chinese stock market is very sensitive to the impact of COVID-19, but the duration of the impact does not last long, which may indicate that the pandemic prevention and control measures implemented in China are effective and timely.

\section{Data Availability}

All the data used in this paper are downloaded from the WIND database, and the details are shown in Table 1.

\section{Conflicts of Interest}

The authors declare that they have no conflicts of interest regarding the publication of this paper.

\section{Authors' Contributions}

Xianbo $\mathrm{Wu}$ and Xiaofeng Hui proposed the research framework together. Xianbo Wu collected the data, finished the computation, and wrote the documents. Xiaofeng Hui provided important guidance and advices during the process of this research.

\section{Acknowledgments}

This research was funded by National Natural Science Foundation of China (Grants nos. 71532004 and 71773024).

\section{References}

[1] J. W. Goodell, "COVID-19 and finance: agendas for future research," Finance Research Letters, vol. 35, Article ID 101512, 2020.

[2] S. Leduc and Z. Liu, "The uncertainty channel of the coronavirus," FRBSF Economic Letter, vol. 7, pp. 1-5, 2020.

[3] A. Sharif, C. Aloui, and L. Yarovaya, "COVID-19 pandemic, oil prices, stock market, geopolitical risk and policy uncertainty nexus in the US economy: fresh evidence from the wavelet-based approach," International Review of Financial Analysis, vol. 70, Article ID 101496, 2020.

[4] B. N. Ashraf, "Stock markets' reaction to COVID-19: cases or fatalities?" Research in International Business and Finance, vol. 54, Article ID 101249, 2020.

[5] D. Zhang, M. Hu, and Q. Ji, "Financial markets under the global pandemic of COVID-19," Finance Research Letters, vol. 36, Article ID 101528, 2020.

[6] F. Aslam, S. Aziz, D. K. Nguyen, K. S. Mughal, and M. Khan, "On the efficiency of foreign exchange markets in times of the COVID-19 pandemic," Technological Forecasting and Social Change, vol. 161, Article ID 120261, 2020.

[7] F. Aslam, T. M. Awan, J. H. Syed, A. Kashif, and M. Parveen, "Sentiments and emotions evoked by news headlines of coronavirus disease (COVID-19) outbreak," Humanities and Social Sciences Communications, vol. 7, p. 23, 2020.

[8] F. Aslam, W. Mohti, and P. Ferreira, "Evidence of intraday multifractality in European stock markets during the recent coronavirus (COVID-19) outbreak," International Journal of Financial Studies, vol. 8, no. 2, p. 31, 2020.

[9] C. T. Albulescu, "COVID-19 and the United States financial markets' volatility," Finance Research Letters, vol. 38, Article ID 101699, 2020.
[10] D. Bakas and A. Triantafyllou, "Commodity price volatility and the economic uncertainty of pandemics," Economics Letters, vol. 193, Article ID 109283, 2020.

[11] A. Zaremba, R. Kizys, D. Y. Aharon, and E. Demir, "Infected markets: novel coronavirus, government interventions, and stock return volatility around the Globe," Finance Research Letters, vol. 35, Article ID 101597, 2020.

[12] D. I. Okorie and B. Q. Lin, "Stock markets and the COVID-19 fractal contagion effects," Finance Research Letters, vol. 38, Article ID 101640, 2020.

[13] M. Akhtaruzzaman, S. Boubaker, and A. Sensoy, "Financial contagion during COVID-19 crisis," Finance Research Letters, vol. 38, Article ID 101604, 2020.

[14] A. S. Baig, H. A. Butt, O. Haroon, and S. Rizvi, "Deaths, panic, lockdowns and US equity markets: the case of COVID-19 pandemic," Finance Research Letters, vol. 38, Article ID 101701, 2020.

[15] M. S. Rizwan, G. Ahmad, and D. Ashraf, "Systemic risk: the impact of COVID-19," Finance Research Letters, vol. 36, Article ID 101682, 2020.

[16] M. Mazur, M. Dang, and M. Vega, "COVID-19 and the March 2020 stock market crash. evidence from S\&P1500," Finance Research Letters, vol. 38, Article ID 101690, 2020.

[17] K. Shehzad, L. Xiaoxing, and H. Kazouz, "COVID-19's disasters are perilous than global financial crisis: a rumor or fact?" Finance Research Letters, vol. 36, Article ID 101669, 2020.

[18] K. Sukcharoen and D. J. Leatham, "Dependence and extreme correlation among US industry sectors," Studies in Economics and Finance, vol. 33, no. 1, pp. 26-49, 2016.

[19] W. Long, Y. Tang, and D. Cao, "Correlation analysis of industry sectors in China's stock markets based on interval data," Filomat, vol. 30, no. 15, pp. 3999-4013, 2016.

[20] H. Qiao, Y. Xia, and Y. Li, "Can network linkage effects determine return? evidence from Chinese stock market," PLoS One, vol. 11, no. 6, Article ID e0156784, 2016.

[21] H. Long, J. Zhang, and N. Tang, "Does network topology influence systemic risk contribution? a perspective from the industry indices in Chinese stock market," PLoS One, vol. 12, no. 7, Article ID e0180382, 2017.

[22] W. Long, L. Guan, J. Shen, L. Song, and L. Cui, “A complex network for studying the transmission mechanisms in stock market," Physica A: Statistical Mechanics and Its Applications, vol. 484, pp. 345-357, 2017.

[23] A. C. Surya, G. Natasha, and G. Natasha, "Is there any sectoral cointegration in Indonesia equity market?" International Research Journal of Business Studies, vol. 10, no. 3, pp. 159172, 2018.

[24] M. Alomari, D. M. Power, and N. Tantisantiwong, "Determinants of equity return correlations: a case study of the Amman Stock Exchange," Review of Quantitative Finance and Accounting, vol. 50, no. 1, pp. 33-66, 2018.

[25] J. Ji, C. Huang, Y. Cao, and S. Hu, "The network structure of Chinese finance market through the method of complex network and random matrix theory," Concurrency and Computation: Practice and Experience, vol. 31, no. 9, Article ID e4877, 2019.

[26] J. G. Poynter, J. P. Winder, and T. Tai, "An analysis of comovements in industrial sector indices over the last 30 years," Review of Quantitative Finance and Accounting, vol. 44, no. 1, pp. 69-88, 2015.

[27] L. E. Kodres and M. Pritsker, "A rational expectations model of financial contagion," The Journal of Finance, vol. 57, no. 2, pp. 769-799, 2002. 
[28] N. Barberis, A. Shleifer, and J. Wurgler, "Comovement," Journal of Financial Economics, vol. 75, no. 2, pp. 283-317, 2005.

[29] D. S. Scharfstein and J. C. Stein, "Herd behavior and investment," American Economic Review, vol. 80, no. 3, pp. $465-479,1990$.

[30] T. C. Chiang and D. Zheng, "An empirical analysis of herd behavior in global stock markets," Journal of Banking \& Finance, vol. 34, no. 8, pp. 1911-1921, 2010.

[31] P. Fiedor, "Networks in financial markets based on the mutual information rate," Physical Review E, vol. 89, no. 5, Article ID 052801, 2014.

[32] P. Fiedor, "Mutual information-based hierarchies on warsaw stock exchange," Acta Physica Polonica A, vol. 127, no. 3a, pp. A-33, 2015.

[33] C. Yang, Y. Shen, and B. Xia, "Evolution of Shanghai stock market based on maximal spanning trees," Modern Physics Letters B, vol. 27, no. 03, Article ID 1350022, 2013.

[34] C. Yang, Y. Chen, W. Hao, Y. Shen, M. Tang, and L. Niu, "Effects of financial crisis on the industry sector of Chinese stock market-from a perspective of complex network," Modern Physics Letters B, vol. 28, no. 13, Article ID 1450102, 2014.

[35] O. Kwon and J.-S. Yang, "Information flow between composite stock index and individual stocks," Physica A: Statistical Mechanics and Its Applications, vol. 387, no. 12, pp. 2851-2856, 2008.

[36] X. Wang and X. Hui, "Mutual information based analysis for the distribution of financial contagion in stock markets," Discrete Dynamics in Nature and Society, vol. 201713 pages, 2017.

[37] X. D. Wang and X. F. Hui, "Cross-sectoral information transfer in the Chinese stock market around its crash in 2015," Entropy, vol. 20, no. 9, pp. 1-14, 2018.

[38] S. Khan, S. Bandyopadhyay, A. R. Ganguly et al., "Relative performance of mutual information estimation methods for quantifying the dependence among short and noisy data," Physical Review E, vol. 76, no. 2, Article ID 026209, 2007.

[39] I. Goldestein and A. Pauzner, "Contagion of self-fulfilling financial crises due to diversification of investment portfolios," Journal of Economic Theory, vol. 119, no. 1, pp. 151-183, 2004. 